\title{
Temporalis muscle transfer in the correction of lagophthalmos due to leprosy
}

\author{
D. SOARES \& M. CHEW \\ Anandaban Leprosy Hospital, P.O. Box 151, Kathmandu, Nepal
}

Accepted for publication 19 May 1996

\begin{abstract}
Summary In the correction of lagophthalmos due to leprosy neuritis temporalis muscle transfer (TMT) is used to provide a motor to assist in lid closure. This study of TMT in 51 eyes was carried out to assess the effectiveness of TMT in achieving lid closure and corneal protection. The average lid gap preoperatively on light closure was $7.3 \mathrm{~mm}$ which was reduced to $3.2 \mathrm{~mm}$ on final follow-up. The average lid gap pre-operatively on tight closure was $5.3 \mathrm{~mm}$ which was reduced to $0.4 \mathrm{~mm}$ at final follow-up. It is possible to train patients with partial or total anesthesia of the cornea in a visual THINK-BLINK reflex. The common complications encountered were ectropion in 6 eyes $(12 \%)$ and ptosis in 3 eyes $(6 \%)$.
\end{abstract}

\section{Introduction}

Lagophthalmos due to paralysis of the orbicularis oculi muscle is found in approximately $5 \%$ of newly diagnosed leprosy patients. ${ }^{5,7}$ This is a result of leprosy neuritis affecting the zygomatic and temporal branches of the facial nerve. Lagophthalmos can lead to exposure keratitis, corneal ulceration, and blindness, especially when there is coexisting corneal anaesthesia.

The main goals of the temporalis muscle transfer (TMT) are to enable the patient to attain closure of the eye, and to prevent loss of vision. This study assesses the degree to which the TMT achieves these goals.

\section{Methods}

Fifty-one TMT operations in 35 patients (27 male and 8 female) were followed up. The operations were performed between 1963 and 1995 (29 since 1992). Sixteen patients had bilateral TMT's, 3 operations were redone due to failure.

Data was collected prospectively on patients operated after 1 January 1994. This consisted of pre-operative, discharge, and follow-up measurements of lid gaps (in $\mathrm{mm}$ ) on light closure (as in sleep) and tight closure (full voluntary musculature), frequency of blinking (blinks/min during spontaneous conversation), voluntary muscle strength of 
orbicularis oculi (graded $0-5$ ), corneal sensation tested with a cottonwool wisp, and visual acuity. Follow-up data for patients operated prior to 1994 was obtained when they presented for review for eye or other problems. Preoperative data for these patients was obtained where available from the physiotherapy assessments.

\section{Technique}

The Johnson ${ }^{2}$ procedure was used in 47 eyes and the Gillies ${ }^{1}$ method was used in 4 eyes. They were all analysed as one group. Although the major points of technique have been described elsewhere, ${ }^{1,2}$ a few salient points need to be made. Usually fascia lata is used as a tendon graft. However if the patient also has a footdrop then a tibialis posterior transfer is also performed at the same session and the plantaris harvested and used as a tendon graft instead.

It is essential that the slips to the lids are tunnelled from about $3 \mathrm{~mm}$ above the lateral canthal margin and are tunnelled from the lateral canthus in the lid margin to avoid postoperative ectropion. ${ }^{4}$ A $6 / 0$ nylon suture is inserted at the middle of the lid to serve as a sling and hold the slip at the lid margin (see Figure 1). This suture is left about $10 \mathrm{~cm}$ long so that at the end of the operation the suture can be taped to the forehead in order to allow the lower eyelid to rest with the lid margin covering the lower third of the cornea.

At the medial canthus the lower slip should be tunnelled around the canthal ligament from above to below (see Figure 2) and attached at maximum tension in order to lift the lower lid as high as possible. The upper slip on the other hand is sutured at low tension so that the upper eyelid at rest sits at the superior edge of the cornea.

The eye is bandaged to prevent excessive swelling for 3 days postoperatively. The sutures at the medial canthus and on the eyelids are removed on day 7 postoperatively and the scalp sutures on day 10. The patient is given a semi-solid diet for 3 weeks and then postoperative physiotherapy and patient education is begun. It is helpful to strengthen the transfer by the use of chewing gum initially and later microcellular rubber. The most important part of postoperative physiotherapy is the development of a THINK-BLINK ${ }^{8}$ reflex. This is done by getting the patient to blink regularly whenever a certain visual stimulus is received. In our hospital we also teach the patient to blink whenever they greet another person.

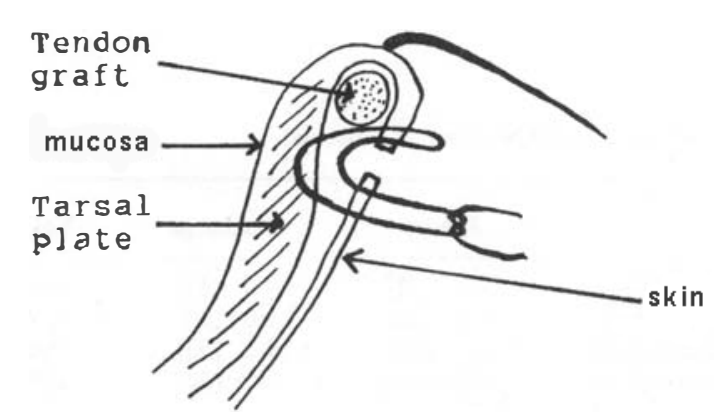

Figure 1. Section through the lid showing a lid suture to prevent ectropion 


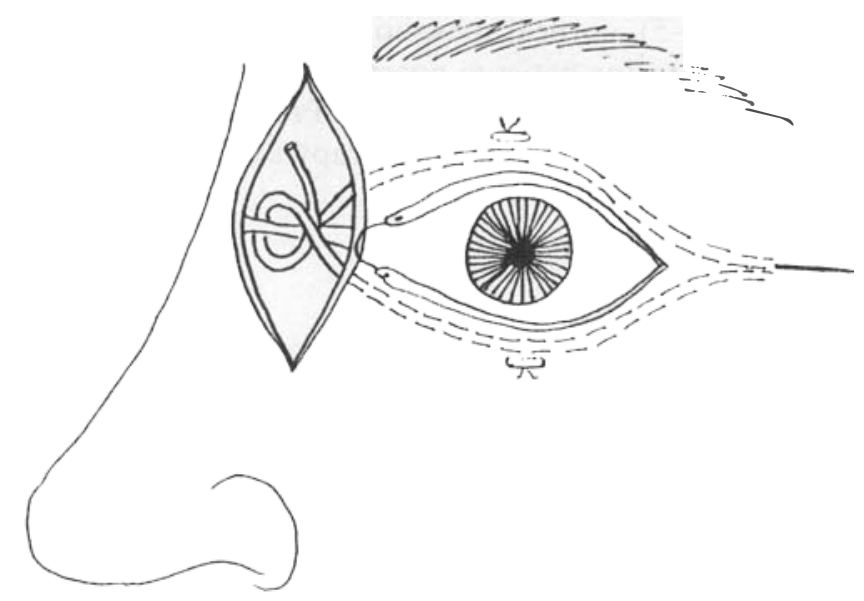

Figure 2. Detail of medial canthus showing attachments of slips.

\section{RESULTS}

The average age was $46 \cdot 3$ years (range 18-78 years). Twenty-eight operations were on the left eye $(55 \%)$, and 23 were on the right $(45 \%)$. The average duration of lagophthalmos prior to operation was 8.0 years. The average duration between operation and follow-up was 7 years 3 months (range 101 days-22 years 7 months). The average duration between operation and discharge was 60 days.

There was no significant difference between preoperative and follow-up measurements of orbicularis oculi muscle strength and corneal sensation.

The average reduction in lid gap on light closure at discharge in 27 patients in whom preoperative and discharge data was available was $4.3 \mathrm{~mm}$ (range $1-8 \mathrm{~mm}$ ). The average reduction in lid gap on tight closure at discharge in 20 patients in whom preoperative and discharge data was available was $4.5 \mathrm{~mm}$ (range $1-10 \mathrm{~mm}$ ).

At discharge 16 out of 27 patients had no lid gap on tight closure with another 5 patients having a lid gap of $1 \mathrm{~mm}$ and 6 patients having a lid gap of $3 \mathrm{~mm}$ or more. At final follow-up 23 out of 30 patients had no lid gap on tight closure with another 4 patients having a lid gap of $1 \mathrm{~mm}$ and only 1 patient having a lid gap of $3 \mathrm{~mm}$.

As data on visual acuity has only been collected prospectively since 1993 it is difficult to reach any meaningful conclusions on whether TMT protects against visual loss. When

Table 1. Average values (Number of patients)

\begin{tabular}{lcccc}
\hline & Pre-op & Discharge & Follow-up \\
\hline Lid gap (light) $\mathrm{mm}$ & $7 \cdot 3(33)$ & $3 \cdot 0(33)$ & $3 \cdot 2(31)$ \\
Lid gap (tight) $\mathrm{mm}$ & $5 \cdot 3(24)$ & $1 \cdot 0(33)$ & $0 \cdot 4(30)$ \\
Blink frequency/min & $6 \cdot 7(21)$ & $9 \cdot 2(27)$ & $7 \cdot 0(27)$ \\
Corneal anaesthesia & $31 \%(36)$ & $39 \%(26)$ & $30 \%(30)$ \\
\hline
\end{tabular}


comparing the groups as a whole there was no significant difference in vision when comparing preoperative, discharge and follow-up patients.

\section{Complications}

In 3 of the 51 eyes, the upper lid slip was too tight causing the inability to adequately open the eye. When it occurs it is usually seen about 3 months postoperatively. This is treated by dividing or Z-lengthening the upper lid slip.

In 6 eyes ectropion was noted at follow-up -in 4 of these (4/17) the operation was performed bef ore 1990. Since 1991 the technique of creating a suture sling to ensure that the slip remained in the lid margin has been used and the complication has only been seen in 2 out of 33 eyes. In this one patient with bilateral transfers the lower slip was not tunnelled from above the lateral canthus but from below the lateral edge leading to lateral ectropion only. When postoperative ectropion is a problem it is necessary to dissect the slip free from the middle of the lid where it has migrated and attach it in the lid margin with 3 or 4 holding sutures as in Figure 1.

\section{Discussion}

Lagophthalmos is a major predisposing factor to loss of vision in people affected by leprosy. When coupled with corneal anaesthesia it has a very poor prognosis.

Correction of lagophthalmos by tarsorrhaphy and/or wedge excision of part of the lid is unsatisfactory. It is cosmetically unacceptable to many patients and often leads to increased irritation of the cornea by the scar on the lid margin. It also fails to provide closure of the eye.

Many workers are fooled into believing a Bell's phenomenon is protective to the eye. The Bell's phenomenon is a voluntary reflex and is lost during sleep thereby causing the cornea to roll forward and be exposed during sleep.

Temporalis muscle transfer provides a good cosmetic result and in the person with corneal sensation provides the effector limb of the reflex arc.

Even in those patients with corneal anaesthesia a THINK-BLINK reflex can be developed with a visual stimulus being the trigger for a voluntary motor action.

Temporalis muscle transfer also serves to reduce the irritation caused to a dry cornea by lagophthalmos. In patients with normal sensation the average blink frequency preoperatively was 20 per minute possibly reflecting corneal irritation. At discharge the blink frequency was 10 per minute. At final follow-up it was 14 per minute.

Training of the patient postoperatively, especially in THINK-BLINK is probably the major determinant of success. In patients with partial or complete corneal anaesthesia blink frequency was much lower preoperatively when compared with patients with normal corneal sensation. However with training in the THINK-BLINK reflex it was possible to improve the spontaneous rate of blinking in these patients (Table 2). This improvement was however lost at final follow-up and suggests the need for ongoing reminders to patients in order to maintain the THINK-BLINK reflex.

In patients with severe lagophthalmos (a lid gap on tight closure of more than $5 \mathrm{~mm}$ ) the TMT reduces the lid gap while maintaining lid mobility so that on light closure as in 
Table 2. Average blink frequency per minute (Number of patients)

\begin{tabular}{lrrr}
\hline & Pre-op & Discharge & Follow-up \\
\hline $\begin{array}{l}\text { Corneal sensation } \\
\text { anaesthetic }\end{array}$ & $2 \cdot 6(7)$ & $8 \cdot 1(10)$ & \\
partial anaes. & $5 \cdot 6(11)$ & $9 \cdot 6(14)$ & $6 \cdot 9(9)$ \\
normal sens. & $20 \cdot 1(3)$ & $10 \cdot 0(20)$ & $14 \cdot 7(6)$ \\
\hline
\end{tabular}

sleep the eye is protected. Some surgeons use TMT only for those patients with sensitive corneas and use tarsorrhaphy for those with anaesthetic corneas. However for the patients with severe lagophthalmos tarsorrhaphy fails to protect the cornea. Even if the patient does a strong blink using the TMT 3 times a minute (average blink frequency at follow-up) then the whole cornea is moistened, whereas with a tarsorrhaphy the inferior aspect of the cornea remains exposed leading to keratitis. It is very difficult to achieve lid closure and protection of the eye using only medial and/or lateral tarsorrhaphy in patients with a lid gap on tight closure exceeding $5 \mathrm{~mm}$. There is a need for long-term follow-up of vision in patients with severe lagophthalmos who have had either TMT or tarsorrhaphy. However in our experience in patients with severe lagophthalmos TMT is the operation of choice.

There is a paucity of long-term follow-up of TMT in the literature. Ranney ${ }^{3}$ in 1973 published a series of 54 TMTs performed on 42 patients. In that series $57 \%$ of eyes achieved full closure. Weber et al. ${ }^{6}$ reported $64 \%$ of eyes with full closure after TMT. In our series $60 \%$ of eyes at discharge and $77 \%$ of eyes at final follow-up achieved full closure.

\section{Conclusion}

TMT is useful in the correction of lagophthalmos due to leprosy especially in those patients with a lid gap on tight closure of greater than $5 \mathrm{~mm}$. It should not only be limited to patients with sensitive corneas as it provides some degree of protection even to those with insensitive corneas. In all patients there was a reduction in lid gap on light and tight closure with complete closure in $76 \%$ of eyes operated on.

\section{References}

1 Andersen JG. Surgical treatment of lagophthalmos in leprosy by the Gillies temporalis transfer. Brit J Plast Surg, 1961; 14: 339-45.

2 Johnson HA. An operation to restore eyelid function lost in leprosy. Int J Lepr, 1965, 33: 89-94.

${ }^{3}$ Ranney, DA \& Furness, MA. Results of temporalis transfer on fifty-four cases of lagophthalmos. Ind J Le pr, 1973, 00: 62-8.

4 Warren G. Facial palsy - a leprosy surgeon's viewpoint. Detailed operation instructions. Aust NZJ Ophthal, 1990, 18: 257-66.

${ }^{5}$ Schipper A, Lubbers WJ, Hogeweg M \& de Soldenhoff R. Disabilities of hands, feet and eyes in newly diagnosed leprosy patients in eastern Nepal. Lepr Rev, 1994, 65: 239-47.

${ }^{6}$ Weber MW, van Soest A, Neff G, Chiang T, Pfau R. Results of surgical procedures for the correction of footdrop and of lagophthalmos due to leprosy. Lepr Rev, 1992, 63: 255-62.

7 van Brakel WH, Khawas IB. Nerve damage in leprosy: an epidemiological and clinical study of 396 patients in west Nepal-Part 1. Definitions, methods and frequencies. Lepr Rev, 1994, 65: 204-21.

8 Watson J. Preventing disability in leprosy patients. The Leprosy Mission International. 